\title{
Solving non-binary convex CSPs in continuous domains
}

\author{
Djamila Sam-Haroud and Boi V. Faltings \\ Artificial Intelligence Laboratory (LIA), \\ Computer Science Department (DI), \\ Swiss Federal Institute of Technology (EPFL), \\ EPFL, Ecublens \\ CH-1015 Lausanne
}

\begin{abstract}
A globally consistent labeling is a compact representation of the complete solution space for a constraint satisfaction problem (CSP). Constraint satisfaction is N P-complete and so is the construction of globally consistent labelings for general problems. However, for binary constraints, it is known that when constraints are convex, path-consistency is sufficient to ensure global consistency and can be computed in polynomial time. We show how in continuous domains, this result can be generalized to ternary and in fact arbitrary n-ary constraints using the concept of (3,2)-relational consistency. This leads to polynomial-time algorithms for computing globally consistent labelings for a large class of numerical constraint satisfaction problems.
\end{abstract}

\section{Introduction}

Many problems, ranging from resource allocation and scheduling to fault diagnosis and design, involve numerical constraint satisfaction as an essential component. These problems often represent complex decision processes where the set of variables and constraints involved is not independent of particular solutions and where relevant information, in the form of active constraints and variables, is revealed only as the task proceeds and decisions are taken. In the case where variables and constraints are numerical, the search space for such problems becomes of an unbounded size; each numerical value may trigger a different active context and thus, potentially lead to a different solution.

Figure 1 shows an example from civil engineering where different values for beam depth and beam span lead to different design options and constraints. Choosing the values of the beam's depth and span within regions 3 or 4 would increase the susceptibility to vibrations and involve installing bridging (lateral reinforcements) for damping the floor (Figure 1,-a-). Choosing these values within region 1,2 or 3 makes it possible to have the ventilation ducts go under the beams while choosing them in region 4 would dictate to make opening in the beams to allow passage of the ducts.

Identifying single point solutions, possibly optimal according to some criterion is the viewpoint adopted by almost all the existing mathematical solvers 
ranging from linear and non-linear programming to numerical analysis and stochastic techniques. Alternatively, consistency techniques offer the possibility of producing a compact description of the space of all solutions by assigning labels (sets of legal values) to individual variables or combination of variables. This is essential for reasoning about design alternatives, as in the example of Figure 1.

While in general, computing globally consistent labeling is NP-hard, recent results [8] show that in the case where constraints are convex, low orders of consistency are equivalent to global consistency. For binary constraints (involving at most 2 variables), it has been shown that 3-consistency (also called pathconsistency and computable in polynomial time) is equivalent to global consistency [8], [2]. However in discrete domains, it has been shown [9] that the generalization of these results to ternary (and higher arity) constraints may involve significantly higher degrees of consistency and thus complexity. In this paper, we show that much more positive results can be obtained in continuous domains.

In fact, we introduce a concept of (3,2)-relational consistency which can be computed in polynomial time and proven equivalent to global consistency for constraint networks containing ternary constraints as well.

We also show how these results can be reliably implemented in practice using an appropriate representation of continuous constraints.

\section{Problem statement}

In this work we consider constraint satisfaction problems in continuous domains. Variable domains are intervals over the reals and constraints are numerical equalities and inequalities of arbitrary types and arities. For practical considerations, the methods developed target problems where both variables and constraints have physical interpretations and can be handled with limited degrees of precision, as is the case in many engineering applications.

A continuous $\operatorname{CSP}(\mathrm{CCSP}),(\mathcal{P}=(V, D, R))$, is defined as a set $V$ of variables $x_{1}, x_{2}, \ldots x_{n}$, taking their values respectively in a set $D$ of continuous domains $D_{1}, D_{2}, \ldots, D_{n}$ and constrained by a set of relations $R_{1}, \ldots, R_{m}$. A domain is an interval of $\Re$ and a relation is defined intensionally by a set of arbitrary equalities and inequalities.

Given a CCSP, we require that for each subset of variables $\left(x_{1}, \ldots x_{k}\right)$, a unique relation $R\left(x_{1} \ldots x_{k}\right)$ exists in the underlying constraint network. In words, each hyper-arc of the constraint network will be labeled by a total constraint [3]. We recall that a total constraint between a set $S$ of variables is given as the region formed by combining all mathematical constraints involving $S$.

We define:

Definition 1. (Convex relation)

Let $\mathcal{P}=(V, C, D)$ be a CCSP. A relation $R\left(x_{1}, \ldots x_{k}\right)$ over $\mathrm{C}$ is convex if it determines a convex solution space in the domain $D_{x_{1}} \ldots \times D_{x_{k}}$, where $D_{i} \in D$. 


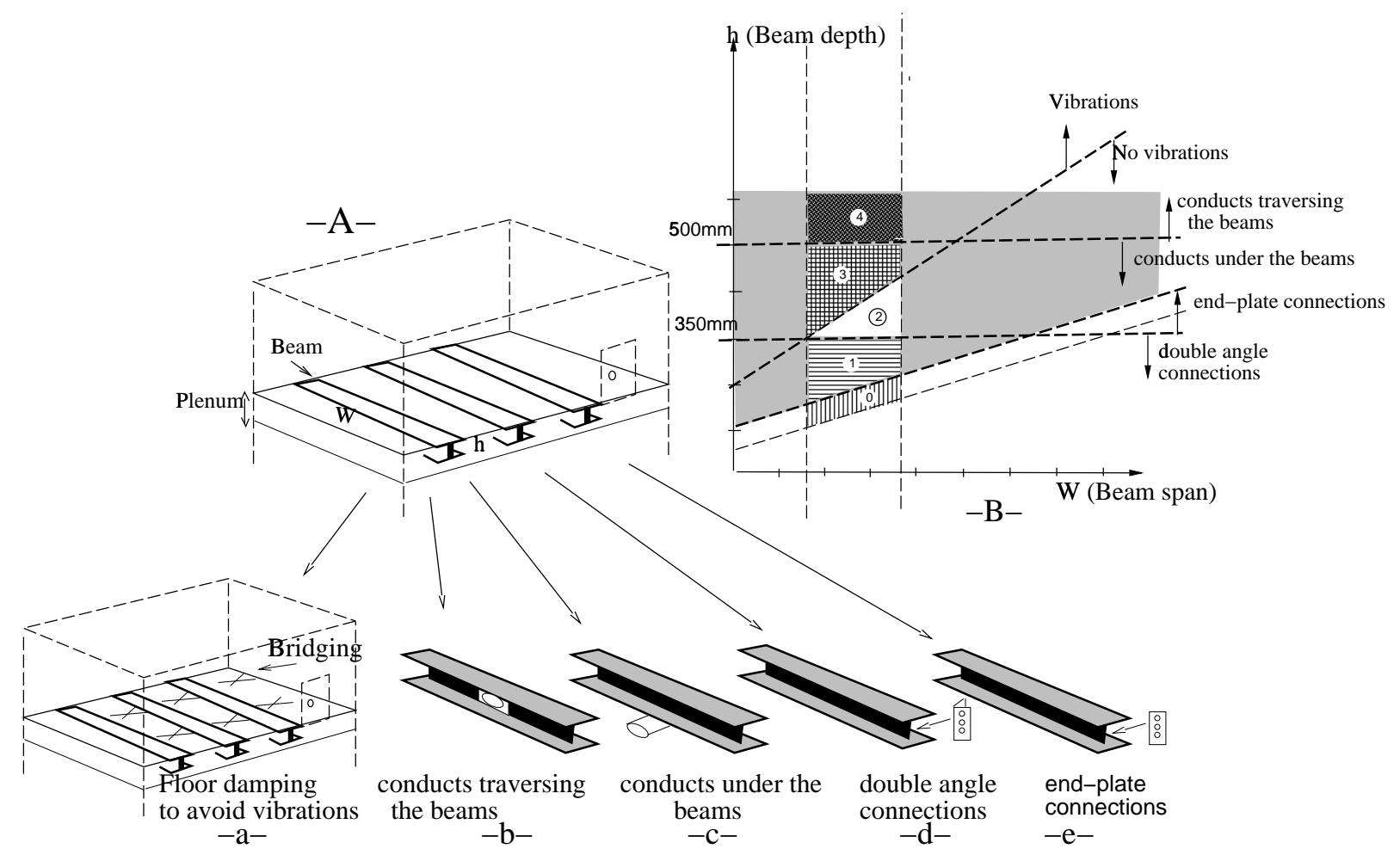

Fig. 1. Many CCSPs are embedded within complex decision processes

A CCSP is called convex when all its relations are convex. In a globally consistent network, any partial consistent instantiation of a subset of variables can be extended to a solution with no backtracking [1], a process which can generally carried out in linear time. For both simple temporal problems and rowconvex discrete problems, it has been observed that convexity of the constraint relations means that path-consistency is sufficient to ensure a globally consistent labeling. This result is proven using Helly's Theorem:

Theorem 2 (Helly). Let $F$ be a finite family of at least $n+1$ convex sets in $R^{n}$ such that every $n+1$ sets in $F$ have a point in common. Then all the sets have a point in common.

Helly's Theorem can be applied to show that for each assignment of $n$ variables $x_{1}, x_{2}, \ldots, x_{n}$, there exists a consistent value which can be assigned to $x_{n+1}$ in the following way. Since the constraint network is binary, the only constraints existing between $x_{1}, \ldots, x_{n}$ and $x_{n+1}$ are individual constraints between $x_{i}, i \in\{1 \ldots, n\}$ and $x_{n+1}$. Since the constraints are convex, every variable $x_{i}$ already assigned constrains $x_{n+1}$ to a single interval. Path-consistency ensures that every pair of such intervals intersect each other. Thus, by Helly's Theorem, 


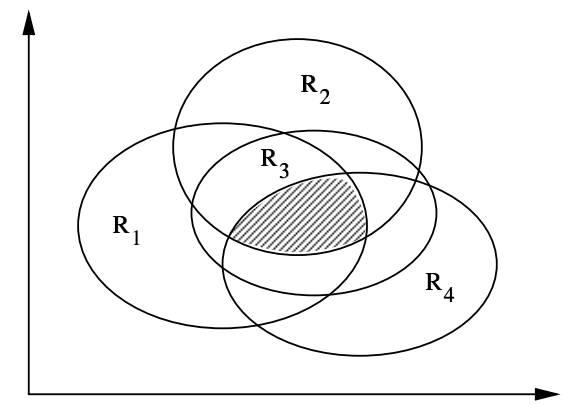

Fig. 2. Helly's Theorem in $\Re^{2}$ : if a finite set of binary convex regions is such that each triplet of regions has a non-null intersection, then the whole set of regions has a non-null common intersection (shaded area)

there must exist a common intersection of all the intervals (i.e., at least one value for $x_{n+1}$ ) which is consistent with all previous assignments, and consequently the assignment can be extended.

\section{Convex n-ary CCSPs}

$\mathrm{N}$-ary continuous and discrete CSPs can be translated into ternary ones without loss of information [4]. To generalize the result from binary discrete networks to ternary discrete CSPs, van Beek and Dechter [9] have introduced the notion of relational path-consistency for discrete problems:

Definition 3 (van Beek \& Dechter). Let $\mathcal{R}$ be a network of relations over a set of variables $X$, and let $\mathcal{R}_{S}$ and $\mathcal{R}_{T}$ be two relations in $\mathcal{R}$, where $S, T \subseteq X$. We say that $\mathcal{R}_{S}$ and $\mathcal{R}_{T}$ are relationally path-consistent relative to variable $x$ iff any consistent instantiation of the variables in $(S \cup T)-\{x\}$, has an extension to $x$ that satisfies $\mathcal{R}_{S}$ and $\mathcal{R}_{T}$ simultaneously. A pair of relations $\mathcal{R}_{S}$ and $\mathcal{R}_{T}$ is relationally path-consistent iff it is relationally path-consistent relative to each variable in $(S \cap T)$. A network is relationally path-consistent iff every pair of relations is relationally path-consistent.

By definition, relational path-consistency guarantees for each set of relations having a variable $x$ in common, that the pairwise intersections of their unary projections over the $x$ axis are non-empty. Helly's Theorem becomes thereby applicable, which results in the following Theorem [9]:

Theorem 4 (van Beek \& Dechter). Let $\mathcal{R}$ be a network of relations that is relationally path-consistent. If there exists an ordering of the domains $D_{1} \ldots D_{n}$ of $\mathcal{R}$ such that the relations are row convex, the network is globally consistent.

Relational path-consistency ensures pairwise non-null intersection of unary projections, with the objective of applying Helly's Theorem in one dimension 


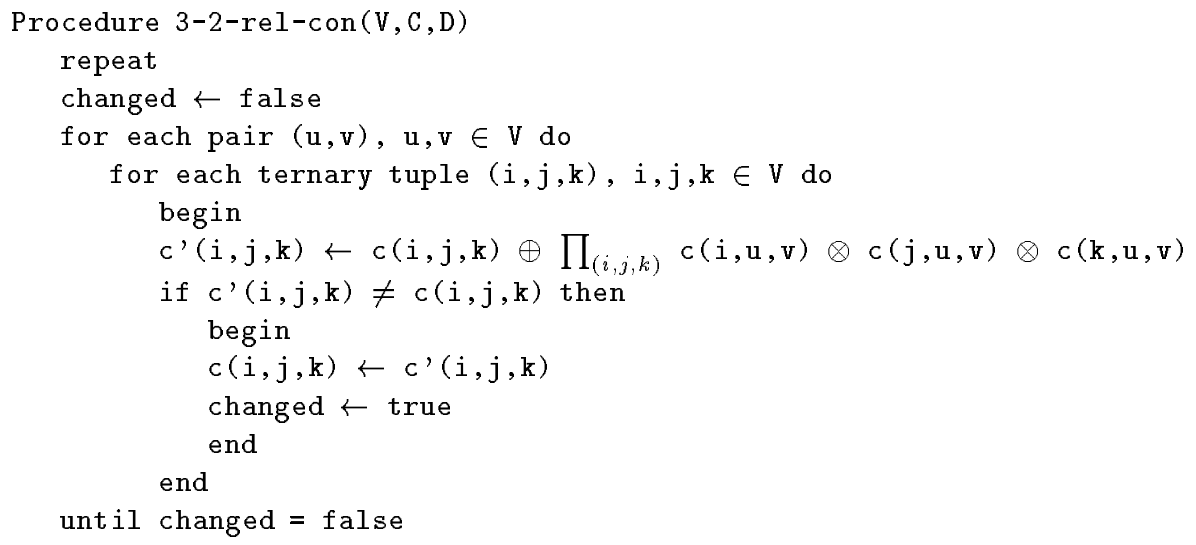

Fig. 3. Algorithm for computing a (3-2)-relationally consistent labeling.

to individual variables. Composing pairs of ternary relations (with a variable in common) results in a relation of arity five. Thus, for global consistency it might be necessary to guarantee that a set of four variables is extensible to a fifth one. But this means that it might be necessary to ensure relational path-consistency for relations of arity four - and recurrently, for relations of unbounded arity, thus possibly engendering an intractable complexity in the most general case.

The alternative generalization we propose is based on the observation that the extensibility of a ternary set of variables to a binary region (rather than to a unary one like for relational path-consistency) does not involve relations with arity greater than 3 and thus removes the causes behind combinatorial explosion. This approach implies that Helly's Theorem must be applied in two dimensions rather than one (see Figure 2). For the case of ternary networks, we introduce the notion of $(3,2)$-relational consistency which guarantees that each triplet of relations, with two variables in common, has a non-null intersection.

Definition 5. (Extension)

Let $\mathcal{P}=(V, C, D)$ be a constraint satisfaction problem. Let $V_{1}$ and $V_{2}$ be subsets of $V . V 1$ has an extension to $V_{2}$ if any consistent instantiation of the variables in $V_{1}$ can be extended to a consistent instantiation of the variables in $V_{1} \bigcup V_{2}$.

Definition 6. ((3,2)-relational consistency)

Let $\mathcal{P}$ be a ternary network of relations over a set of variables $\mathrm{X}$. Let $R_{I_{1}}\left(x_{1}, u, v\right)$, $R_{I_{2}}\left(x_{2}, u, v\right)$ and $R_{I_{3}}\left(x_{3}, u, v\right)$ be three relations of $N$ which share two variables $\mathrm{u}$ and $\mathrm{v}$, where $\mathrm{u}$ might be identical to $\mathrm{v} . R_{I_{1}}, R_{I_{2}}$ and $R_{I_{3}}$ are $(3,2)$-relationally consistent relative to $\{u, v\}$ if and only if any consistent instantiation of the 3 variables in $x_{1}, x_{2}, x_{3}$ has an extension to $\{u, v\}$ that satisfies $R_{I_{1}}, R_{I_{2}}$ and $R_{I_{3}}$ simultaneously.

Since (3,2)-relational consistency only requires labels between at most three variables, it does not add to the arity of a ternary constraint network. Provided 
that each binary projection is convex, $(3,2)$-relational consistency enables the application of Helly's Theorem in two dimensions. However, Helly's Theorem only guarantees that each pair of variables has a non-empty domain. It remains to show that the constraints on each individual variable are also non-empty.

The simple algorithm of Figure 3 terminates with a set of $(3,2)$-relational consistent set of labels.

This algorithm takes as input a ternary CCSP, $\mathcal{P}=(V, C, D)$ where $V$ is the set of variables, $C$ the set of constraints, and $D$ is the set of variable domains. $c$ denotes the label of a relation in $C$. $R$ denotes relations in $C$.

Using Helly's Theorem in two dimensions we can state the following result:

Theorem 7. For any convex ternary network $\mathcal{P}$, (3,2)-relational consistency will either decide that $\mathcal{P}$ is inconsistent, or else compute an equivalent globally consistent labeling of $\mathcal{P}$, in time $O\left(n^{5}\right)$ where $n$ is the number of variables of $\mathcal{P}$.

Informally, the proof consists of the following steps:

1. we first prove that when applied to a ternary network $\mathcal{P}$, the algorithm for $(3,2)$-relational consistency results in an empty network if a given pair of variables has an empty label,

2. in order to show that non-empty labels on each pair of variables implies global consistency, we introduce a binary dual representation of the original ternary problem. This dual representation, by the fact that it is binary, makes it easier to show how an instantiation process can be carried out backtrack free when binary labels are non-empty,

3 . the dual representation is shown to be globally consistent and equivalent to the primal one.

The binary dual representation of a ternary network $\mathcal{P}(V, C, D)$ is a binary network, $\mathcal{P}_{d}\left(V_{d}, C_{d}, D_{d}\right)$, such that:

$-V_{d}=\left\{\alpha_{1}, \ldots \alpha_{m}\right\}$. A variable of $V_{d}, \alpha_{j}$, represents a pair of variables in the original network (an element $\left(x_{(j, 1)}, x_{(j, 2)}\right)$ of $V^{2}$ so that $\left.x_{(j, 1)} \neq x_{(j, 2)}\right)$

- a domain of $D_{d}$ is an element of $D^{2}$

- a relation between two variables, $\alpha_{u}$ and $\alpha_{v}$, of $V_{d}$ is the relation $R\left(x_{(u, 1)}, x_{(u, 2)}, x_{(v, 1)}, x_{(v, 2)}\right)$ resulting from the composition of the relations between $x_{(u, 1)}, x_{(u, 2)}, x_{(v, 1)}, x_{(v, 2)}$ in the original problem $\mathcal{P}$.

In the following, the fact that each pair of variables has a non-empty label will be referred to as the binary-extensibility property:

Definition 8. (Binary-extensibility)

Let $\mathcal{P},(V, C, D)$, be a network of relations. $\mathcal{P}$ is said to be binary-extensible if any subset of $V$ 's variables has an extension to any pair of variables of $\mathrm{V}$.

We now present the intermediate results needed for stating Theorem 7 along with sketches of their proofs. 
Lemma 9. Let $\mathcal{P}^{\prime}$ be a (3,2)-relationally consistent ternary network and let $\mathcal{P}_{d}^{\prime}$ be its dual representation. The following propositions are verified:

$i$. each partial solution of $\mathcal{P}^{\prime}$ corresponds to a partial solution of $\mathcal{P}_{d}^{\prime}$

ii. each partial solution of $\mathcal{P}_{d}^{\prime}$ corresponds to a partial solution of $\mathcal{P}^{\prime}$

This results follows immediately from the definition of the dual network representation.

Corollary 10. Let $\mathcal{P}^{\prime}$ be a (3,2)-relationally consistent ternary network and let $\mathcal{P}_{d}^{\prime}$ be its dual representation. $\mathcal{P}^{\prime}$ is equivalent to $\mathcal{P}_{d}^{\prime}$.

$\mathcal{P}^{\prime}$ and $\mathcal{P}_{d}^{\prime}$ are equivalent in the sense that each solution of the first network is also a solution of the latter one, and vice versa. Since Lemma 9 is stated for arbitrary partial instantiations it also hold for a global instantiation.

Lemma 11. Let $\mathcal{P}$ be a ternary network of relations, $\mathcal{P}^{\prime}$ be its (3,2)-relationally consistent counterpart and $\mathcal{P}_{d}^{\prime}$ be the dual representation of $\mathcal{P}^{\prime}$. If $\mathcal{P}$ has no solution, $\mathcal{P}_{d}^{\prime}$ is empty.

Sketch of proof. An inconsistent network $\mathcal{P}$ is a fortiori not binary-extensible (i.e there exists at least one pair of variables with an empty label). Since the algorithm for $(3,2)$-relational consistency computes the closure of $\mathcal{P}$ with respect to binary-extensibility, it will therefore necessarily results in an empty $(3,2)$-relationally consistent representation $\mathcal{P}^{\prime} . \mathcal{P}^{\prime}$ being equivalent to $\mathcal{P}_{d}^{\prime}$ (Corollary 10$), \mathcal{P}_{d}^{\prime}$ is also empty $\square$.

Lemma 12. Let $\mathcal{P}$ be a ternary network of relations, $\mathcal{P}^{\prime}$ be its (3,2)-relationally consistent counterpart and $\mathcal{P}_{d}^{\prime}$ be the dual representation of $\mathcal{P}^{\prime}$. If $\mathcal{P}_{d}^{\prime}$ is nonempty, $\mathcal{P}_{d}^{\prime}$ is globally consistent.

Sketch of proof. Suppose that $k-1$ variables of $\mathcal{P}_{d}^{\prime}$ have been consistently instantiated. Using Helly's Theorem in two dimensions, we first show [5] that in the case where the relations of $\mathcal{P}_{d}^{\prime}$ are not empty, each consistent instantiation of three variables in $\mathcal{P}_{d}^{\prime}$ can be consistently extended to a fourth one. This guarantees that each subset of three $\mathcal{P}_{d}^{\prime}$ 's relations, $R\left(\alpha_{k}, \alpha_{a}\right), R\left(\alpha_{k}, \alpha_{b}\right), R\left(\alpha_{k}, \alpha_{c}\right)$, (where $(a, b, c) \in[1 . . k-1])$ has a non-null projection over $\alpha_{k}$. Since each relation $\prod_{\alpha_{k}} R\left(\alpha_{k}, \alpha_{i}\right)$ is a convex, non-empty region of $\Re^{2}$ (variables $\alpha_{i}, i \in$ $[1 . . k-1]$ are instantiated), Helly's Theorem is applicable and guarantees that $\prod_{\alpha_{k}}^{i:[1 . . k-1]} R\left(\alpha_{k}, \alpha_{i}\right) \neq \emptyset$. This means that $\alpha_{k}$ can be instantiated consistently. This result holds for an arbitrary $k$, hence $\mathcal{P}_{d}^{\prime}$ is globally consistent $\square$.

Theorem 7 follows immediately from Lemmas 9,11 and $12 . \mathcal{P}$ being a ternary convex network of relations, $\mathcal{P}^{\prime}$ its $(3,2)$-relationally consistent counterpart and $\mathcal{P}_{d}^{\prime}$ the dual representation of $\mathcal{P}^{\prime}$, Lemma 9 guarantees the equivalence of $\mathcal{P}^{\prime}$ and $\mathcal{P}_{d}^{\prime}$, Lemma 11 ensures that an inconsistent $\mathcal{P}$ results in an empty $\mathcal{P}_{d}^{\prime}$ (and hence $\mathcal{P}^{\prime}$ ) representation, and finally, Lemma 12 guarantees that if a solution exists, $\mathcal{P}_{d}^{\prime}$ (and hence $\mathcal{P}^{\prime}$ ) is globally consistent. 
Complexity. The number of relations checked for binary-extensibility is initially in $O\left(n^{3}+n^{2}\right)=O\left(n^{3}\right)$. Each time a relation is modified, $O\left(n^{2}+n\right)=O\left(n^{2}\right)$ new (3,2)-relational compositions are computed. The global time complexity of $(3,2)$-relational consistency is therefore $O\left(n^{5}\right) \square$.

Given that $k$ variables $\left\{x_{1}, x_{2} \ldots x_{k}\right\}$ of $\mathcal{P}$ have already been instantiated, finding a value for a third variable $x_{k+1}$ is always possible: it amounts to finding a value, in $\mathcal{P}_{d}^{\prime}$, for a node $\left(x_{k+1}, x_{j}\right), j=[1 . . k]$. A possible backtrack-free instantiation procedure for deriving the solutions of $\mathcal{P}$ would be as follows:

1. Choose a value $X_{1}$ of $x_{1}$ that satisfies $R\left(x_{1}\right)$

2. For $i \leftarrow 2$ to $n$ do

3. $\quad I_{i} \leftarrow \bigcap_{j: 1 . . i-1} \prod_{x_{i}} R\left(x i, X_{1}, X_{j}\right)$

4. $\quad X_{i} \leftarrow$ choose a value for $x_{i}$ in $I_{i}$

\section{$4 \quad$ Partial and directional convexity properties}

Constraint convexity is a rather strong condition, but it turns out that weaker forms of convexity are often sufficient to satisfy the conditions of a globally consistent labeling.

Partially convex binary CCSPs We introduce in $[4,5]$ a new category of partial convexity called $(x)$-convexity for binary relations. This property is more restrictive than path and simple connectivity but guarantees that convexity is maintained while enforcing relational consistency.

Definition 13. ((x)-Convexity [4])

Let $R$ be a binary relation defined by a set of algebraic or transcendental constraints on two variables $x_{1}, x_{2} . R$ is said to be $x_{k}$-convex in the domain $D_{x_{k}}$ if for any two points $q_{1}$ and $q_{2}$ of $r$ such that the segment $\overline{q_{1} q_{2}}$ is parallel to $x_{k}$, $\overline{q_{1} q_{2}}$ is entirely contained in $r$.

A network is said to be (x)-convex if each of its relations $R(x, y)$ is (x)-convex.

(x)-convexity guarantees the convexity of any unary projection of a given relation [5]. This allows the formulation of the following result [4].

Theorem 14. A binary CCSP which is (x)-convex and path-consistent is minimal and decomposable

The $(x)$-convexity property is non-conservative with respect to intersection. Hence, the global consistency property stated by Theorem 14 is only guaranteed for the a posteriori network computed by path-consistency.

Directional (x)-convexity In the case where a discrete network does not satisfy the row-convexity property, van Beek shows that directional row-convexity remains a useful property for obtaining backtrack-free solutions. Similar results generalizes to the case of (x)-convex relations. The following Theorem states [4] that a partial (x)-convexity of the network is sufficient to ensure that a solution can be determined without backtracking. 
Theorem 15. Let $\mathcal{N}$ be a path-consistent binary constraint network. If there exists an ordering of the variables $x_{1}, \ldots x_{n}$ such that each relation $R\left(x_{i}, x_{j}\right)$ of $\mathcal{N}$ with $1 \leq j \leq i$, is $\left(x_{i}\right)$-convex, then a consistent instantiation can be found without backtracking.

Directional row-convexity imposes ordering conditions on both variables and variable domains. Since discrete CSPs do not have the strictly ordered domains characterizing continuous CSPs, the fact that a constraint network is row-convex can sometimes be hidden. This is obviously not the case for continuous CSPs concerning (x)-convexity.

Partially convex n-ary relations Similarly to the case of binary constraints, a less restrictive convexity condition can be defined for n-ary constraints. We first propose the following generalization of the $(x)$-convexity property:

Definition 16. $\left(\left(x_{1}, \ldots x_{k}\right)\right.$-Convexity)

Let $R$ be an n-ary relation between $n$ variables $x_{1} \ldots x_{n}$. $R$ is said to be $\left(x_{1}, \ldots x_{k}\right)$ convex in the domains $D_{1} \times \ldots D_{x_{k}}$ if for any two points $q_{1}$ and $q_{2}$ of $r$, such that the segment $\overline{q_{1} q_{2}}$ is on a plane parallel to $x_{1} \ldots \times x_{k}, \overline{q_{1} q_{2}}$ is entirely contained in $r$

Informally, this means that a relation is $\left(x_{1}, \ldots x_{k}\right)$-convex if any sub-projection over the subset $\left(x_{1}, \ldots x_{k}\right)$ yields a convex $k$-ary region. In the case of networks of arity $r$, the composition of two maximal arity constraints having at least one variable in common, results in a relation of arity $2 r-1$. In analogy to the case of ternary networks, we observe that the extension of an r-ary set of variables to a region of arity $r-1$ does not involve relations with arity greater than $r$. To apply Helly's Theorem we must introduce the notion of $(\mathrm{r}, \mathrm{r}-1)$-relational consistency which guarantees that each set of $r$ relations having $r-1$ variables in common has a non-null intersection:

Definition 17. ((r,r-1)-relational consistency) Let $\mathcal{P}$ be a network of relations over a set of variables $\mathrm{X}$, of arity $r$. Let $R_{I_{1}}\left(x_{1}, y_{1}, \ldots, y_{r-1}\right), \ldots R_{I_{r}}\left(x_{r}, y_{1}, \ldots, y_{r-1}\right)$ be $r$ relations of $N$ sharing the $\mathrm{r}-1$ variables $\left\{y_{1}, \ldots y_{r-1}\right\}$. The relations are (r,r-1)-relationally consistent relative to the shared variables if and only if any consistent instantiation of the variables in $\left\{x_{1}, \ldots, x_{r}\right\}$ has an extension to $\left\{y_{1}, \ldots y_{r-1}\right\}$ that satisfies all relations simultaneously. The network $\mathcal{P}$ is relationally (r,r-1) consistent if and only if all relations are $(\mathrm{r}, \mathrm{r}-1)$-consistent with respect to all subsets of shared variables.

Hence, the following generalization of Theorem 14 can be proposed:

Theorem 18. Let $\mathcal{P}$ be a constraint network of arity $r$ at most, $\left(x_{1}, \ldots, x_{r-1}\right)$ convex. If $\mathcal{P}$ is $(r, r-1)$-relationally consistent, then it is globally consistent.

Proof. The proof is similar to the one given for Theorem 7 . In the general case, the nodes in the binary dual representation of $\mathcal{P}$ represent $(r-1)$-ary subsets of $\mathcal{P}$ 's variables. 
Directional (x,y)-convexity In the proof of Theorem 7, convexity of the constraints is used only in the application of Helly's Theorem to ensure extensibility of partial solutions of the dual network. Here, it would be sufficient to have convexity hold only in two of the three dimensions involved in the constraint. Thus, we have the following Theorem:

Theorem 19. Let $\mathcal{P}$ be a (3,2)-relationally consistent ternary constraint network. If there exists an ordering of the variables $x_{1}, \ldots x_{n}$ such that for any $i, j, k: 1 \leq i<j \leq k \leq n, R\left(x_{i}, x_{j}, x_{k}\right)$ is $\left(x_{j}, x_{k}\right)$-convex, then the network is globally consistent and a consistent instantiation can be found without backtracking.

Sketch of proof. According to Helly's Theorem in two dimensions, the fact that each ternary relation $R\left(x_{i}, x_{j}, x_{k}\right)$ is $\left(x_{j}, x_{k}\right)$-convex guarantees that the binary relations $R\left(x_{j}, x_{k}\right)$ derived from the problem are non-empty $\left(R\left(x_{j}, x_{k}\right)=\right.$ $\left.\bigcap_{j: i . . k} \prod_{x_{i}} R\left(x_{i}, x_{j}, x_{k}\right)\right)$. By construction, these binary relations are convex and have non-null pairwise intersections. Consequently, a similar argument as the one given for the proof of Theorem 7 hold and instantiation can be carried out backtrack-free $\square$.

\section{$5 \quad$ Exploiting convexity in practice}

In discrete domains, relations are represented simply as enumerations of values or value combinations. In continuous domains, sets of individual values are often compact and can be represented by one or a small collection of intervals. However, representing and manipulating labels of several variables, as it is necessary for implementing algorithms for higher degree of consistency than two is more involved as they may be complex geometric shapes.

Constraint representation In [4], we propose to represent numerical constraints using $2^{k}$-trees (a hierarchical representation of space commonly used in vision and spatial reasoning [7]). The $2^{k}$-trees representation of constraints is based on the observation that in most practical applications each variable takes its values in a bounded domain (bounded interval) and there exists a maximum precision with which results can be used.

Provided that these two assumptions hold, a relation defined by inequalities can be approximated by carrying out a hierarchical binary decomposition of its solution space into $2^{k}$-trees (quadtrees for binary relations, octrees for ternary ones etc...) (see Figure 4). In order to provide a unified framework for handling both inequalities and equalities, we propose in [4] to translate equalities into a weaker form called toleranced equalities [6]: the final grey nodes of the $2^{k}$ tree decomposition for an equality constraint are replaced by white nodes. This amounts to replacing each equality by two inequalities close to each other and is acceptable in practice as long as a the results can be identified with a limited precision. 


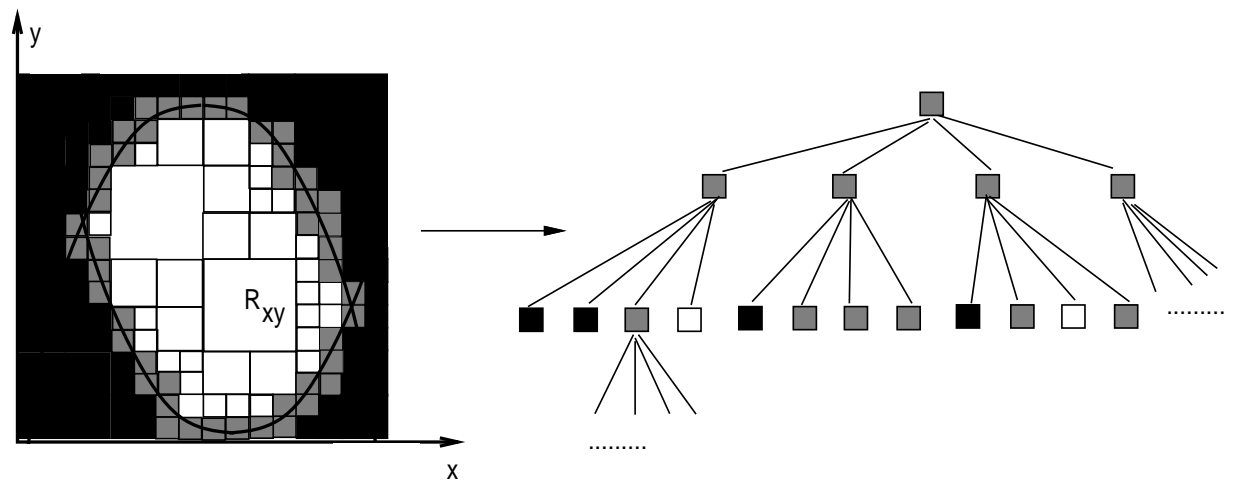

Fig. 4. A continuous relation can be approximated by carrying out a hierarchical binary decomposition of its solution space into a $2^{k}$-tree where: white nodes represent completely legal solution regions, grey nodes partially legal and partially illegal regions and black nodes completely illegal ones

Using this discretized representation, the sets of feasible value combinations can be interpreted and manipulated explicitly. The main advantage is that the use of complex numerical tools for solving sets of simultaneous constraints (stated implicitly by their mathematical expressions) can be avoided. The approximate solution region defined by a set of several constraints is constructed by projecting, composing and intersecting their individual $2^{k}$-tree representations. These operations on $2^{k}$-trees are easy to implement and extensively studied in computer vision, computer graphics and image processing. Moreover, constructing the $2^{k}$-tree representation for a single constraint only requires evaluating an individual constraint equation at certain points in the space [6]. As we show in $[4,5]$, the explicit handling of solution regions using $2^{k}$-trees conveys a simple implementation for path- and higher degrees of consistency in continuous domains.

Correctness of the representation A $2^{k}$-tree representation can be interpreted as providing two different approximations for a feasible region:

- the inner content approximation, $\mathcal{I}(\mathcal{S})$, is given by the white nodes (interior nodes) and is entirely enclosed within the solution space. Since all values within $\mathcal{I}(\mathcal{S})$ are consistent, it is a sound approximation. However, some solutions maybe missing from this representation,

- the closest outer content approximation, $\mathcal{O}(\mathcal{S})$, is given by the union of the white and grey nodes (interior nodes $\cup$ boundary nodes). This approximation is guaranteed to contain all solutions, but it may be not sound since the grey nodes contain inconsistent values.

With regard to equalities, remember that this method only allows toleranced equalities and soundness will only hold with respect to these tolerances. For the 
initial $2^{k}$-tree representations of individual constraints, we can always guarantee that $\mathcal{I}(\mathcal{S})$ and $\mathcal{O}(\mathcal{S})$ are as close as possible to the actual solution region. However, constructing total constraints and enforcing consistency involves composition and intersection operations on constraints.

Letting $\mathcal{S}_{1} \oplus \mathcal{S}_{2}$ denote the solution space resulting from the intersection of $\mathcal{S}_{1}$ and $\mathcal{S}_{2}$. We can show the following properties (see [5]):

- The inner content approximation is exact with respect to intersection: $\mathcal{I}\left(\mathcal{S}_{1}\right) \oplus \mathcal{I}\left(\mathcal{S}_{2}\right)=\mathcal{I}\left(\mathcal{S}_{1} \oplus \mathcal{S}_{2}\right)$

- The outer content approximation may contain spurious nodes after intersection: $\mathcal{O}\left(\mathcal{S}_{1} \oplus \mathcal{S}_{2}\right) \subseteq \mathcal{O}\left(\mathcal{S}_{1}\right) \oplus \mathcal{O}\left(\mathcal{S}_{2}\right)$

For composition, it is possible to show that the projection of a constraint into a higher-dimensional space is exact for both inner and outer approximations. Therefore, the $\mathcal{I}(\mathcal{S})$ representation of total constraints is exact, even after executing consistency algorithms. This means that it is both sound (not containing any spurious inconsistent values) as well as maximal in the sense that there is no larger sound $\mathcal{I}(\mathcal{S})$ approximation, for a given precision. On the other hand, the $\mathcal{O}(\mathcal{S})$ representation computed by logical combination of simultaneous constraints is complete but not sound with respect to the minimal enclosing approximation $\mathcal{O}(\mathcal{S})$ - spurious grey nodes can be created by intersections.

$\mathbf{2}^{k}$-trees and convexity Since the $2^{k}$-tree decomposition generates stepwise approximations of the boundaries, convexity is obviously not preserved in the strict mathematical sense. In [5], we show that when the resolution chosen is insufficient, situations may occur where a connected solution space is represented by disconnected or even empty $\mathcal{I}(S)$ representation. However, these limitations are compensated by the fact that:

- the $\mathcal{I}(S)$ representation of a convex solution space can be empty or disconnected only when the solution of the CCSP falls within the limit of resolution chosen for the $2^{k}$-tree representation. This situation is therefore restricted to limit cases,

- when the $\mathcal{I}(S)$ representation of a convex solution space is disconnected, a single additional level of decomposition is then sufficient to make the representation connected again.

Hence, if a disconnection occurs (limit cases), it is consequently possible either to resort to further refinements of the quadtrees or to neglect the solution region within the disconnected area-considering that its identification requires a precision having no significance for the application. Moreover, a particular class of minimal convexity deficiencies can be identified which precludes the risk of disconnection (see [5]). The $2^{k}$-trees having minimal deficiencies of this type are said to be convex.

Checking for convexity When constraints are approximated using quadtrees, we show in [5] that the (x)-convexity property can checked for in $O\left(N \log _{4}(N)+\right.$ 
$2^{D_{y} / \varepsilon}$ ) where $N$ is the number of feasibility nodes, $D_{y}$ is the domain size of variable $x$ (i.e. interval length) and $\varepsilon$ the minimal interval length of $x$ in the quadtree decomposition. Similarly, convexity can be checked for in $O\left(2 . N \log _{4}(N)+2^{\frac{D}{\varepsilon}+1}\right)$ where $N$ is the number of feasibility nodes, $D$ is the maximal domain size in the quadtree (i.e. interval length) and $\varepsilon$ the minimal interval length in the quadtree decomposition (for a fixed precision, this complexity is $O\left(N \log _{4}(N)\right)$. Finally, using analog procedures, the $\left(x_{1}, x_{2}\right)$-convexity property, useful for solving ternary problems, can be checked for in $O\left(N+N \log _{4}(N)\right)$, for a fixed precision. These simple convexity checking procedures examine exhaustively the boundary nodes of the $2^{k}$-tree representations. For detailed descriptions we refer the reader to [5].

Comparison with the discrete case- $2^{k}$-trees and backtrack-free search It is worth mentioning that the results on n-ary constraints (see section 3 ) are not directly transferable to discrete domains. Ensuring backtrack-free search in ternary constraints requires convexity conditions to hold in $\Re^{2}$ rather that $\Re$. We have shown that $(3,2)$-relational consistency is equivalent to global consistency, but the backtrack-free instantiation might require refining the resolution of different variables. This is possible in continuous domains, but not possible if we use a continuous domain to represent a discrete problem.

Consider the following example where two matrices representing discrete relations are understood as showing convex solution regions. When we intersect the two regions, the result is:

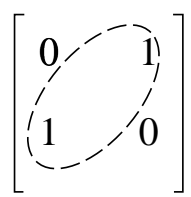<smiles>CCCOCCOC</smiles>

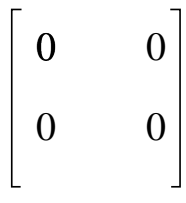

i.e. the intersection has been lost as it is smaller than the resolution limit. In a continuous problem, we can now refine the resolution to make this problem go away, and continue with the instantiation. But in a discrete problem, we do not have this possibility as the maximum resolution is fixed. A path-consistent labeling does not guarantee that we can in fact successfully complete a backtrackfree instantiation without need for increasing the resolution, and hence does not guarantee global consistency in a discrete problem where this possibility does not exist.

On the other hand, extending the row-convexity property to $2 \mathrm{D}$, so that Helly's Theorem can ensure the binary extensibility condition, would dictate that each discrete ternary relation yields a universal matrix (with 1s only) as binary projection, which is probably too restrictive for practical use.

\section{Example}

We now sketch out how the introductory example of Figure 1 is solved using our method. In this example, four main independent variables, beam $\operatorname{depth}\left(H_{b}\right)$, slab 

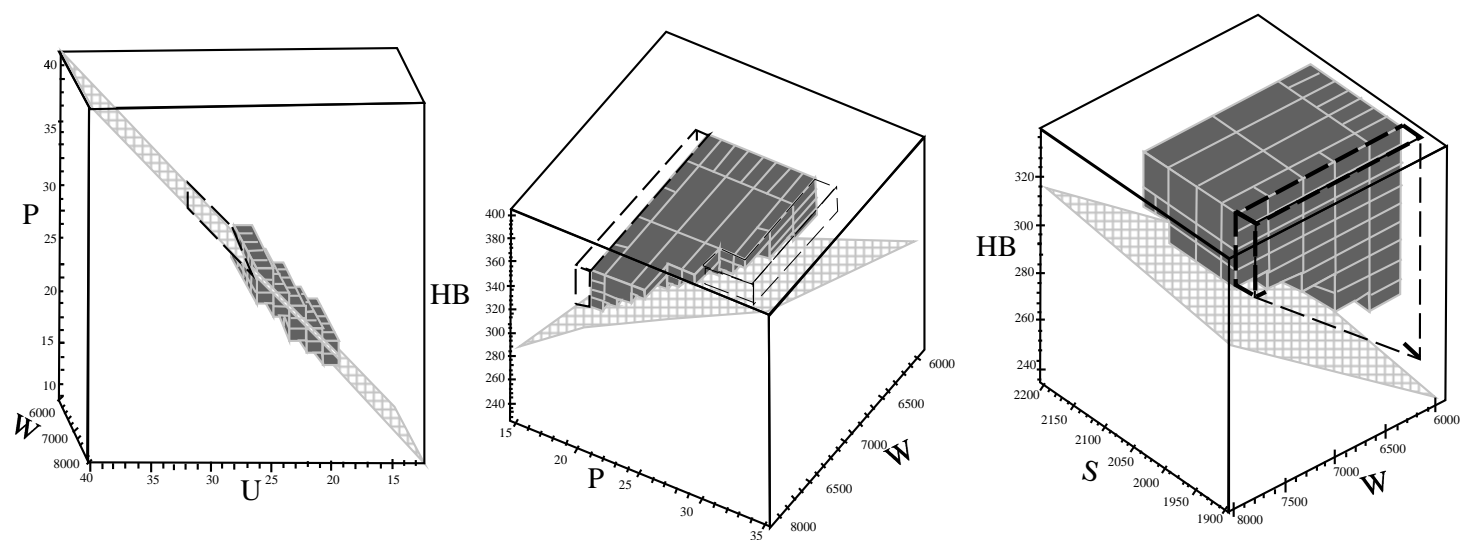

Fig. 5. Three constraints from the steel structure problem. The areas within the dashed lines are those removed while enforcing global consistency

thickness $\left(H_{s}\right)$, beam span(W) and beam spacing(S) are linked together through the following non-linear constraints:

$$
\begin{aligned}
H_{s} & >137.70-8633.10^{-3} S+5511.10^{-8} S^{2}-835810^{-10} S^{3} \\
H_{b} & >41.383\left(\frac{1.1 * 2.35 * p * W^{2}}{10^{6} C * f_{y}}\right)^{0.3976} \\
p & =1.3\left[\frac{\left(P_{\text {sw }(\text { slab })}+P_{d l}\right) * S}{1000}+0.000074\left(\frac{W}{24}\right)^{1.5161}\right] \\
H_{b} & >\left[\frac{P_{l l s t} * S}{1000} \cdot \frac{D * 350 * W^{3}}{0.1545 * 384 * E_{s}}\right]^{0.2839}
\end{aligned}
$$

Traditionally, an engineer works through these equations hierarchically; at no time is the complete solution set known. Exploration of possible solutions is done point by point according to the experience of the designer.

Using our system, a prototype Lisp implementation calculates the globally consistent solutions in $\sim 1800$ seconds on a Silicon Graphics Indigo with an R4000 processor. Figure 5 shows a set of constraints derived from the problem after global relaxation: the areas within the dashed lines are those removed by $(3,2)$-relational consistency. ( $u$ is an intermediate variable used when transforming the original problem into a ternary one, $u=318.10^{-8} H_{s} * S+0.0054$ ).

This shows that the problem admits in fact a large space of potential solutions, of which current mathematical methods only find a single one.

\section{Conclusion}

Convexity has been shown to be a useful property for efficiently solving binary discrete and temporal constraint satisfaction problems. In this paper, we 
propose a generalization of these results to continuous constraints of arbitrary arities. While in discrete domains it has been shown that the generalization of the results on convexity to ternary (and higher arity) constraints may pose complexity problems, we introduce a concept of $(3,2)$-relational consistency which can be computed in polynomial time and proven equivalent to global consistency for constraint networks containing ternary constraints as well. Since n-ary constraint problems can always be transformed into equivalent ternary ones, these results guarantee polynomial-time solution for a large class of continuous n-ary problems. We also show how these results can be exploited in practice. The applicability condition of these results is that a limited precision must exist under which the results have no significance. This condition holds for almost all the engineering applications manipulating physical entities.

\section{Acknowledgment}

We would like to thank Sylvie Boulanger (Steel Structure lab., EPFL) for interesting discussions related to civil engineering, Peter van Beek (University of Alberta, CA), and Gaston Gonnet (ETHZ) for many helpful comments on this work, as well as the Swiss National Science Foundation for sponsoring this research under contract No.5003-034269.

\section{References}

1. Dechter R. From local to global consistency. In Proceedings of the 8th Canadian Conference on $A I, 1990$.

2. Dechter R., Meiri i., and Pearl J. Temporal constraint networks. In Artificial Intelligence 49(1-3), 1990.

3. Faltings B. Arc consistency for continuous variables. In Artificial Intelligence 65(2), 1994.

4. Haroud D. and Faltings B.V. Global consistency for continuous constraints. In Alan Borning, editor, Lecture notes in computer Science 874: Principles and Practice of constraint programming. Springer Verlag, 1994.

5. Sam-Haroud D. Constraint consistency techniques for continuous domains. PhD thesis, Ecole Polytechnique Fédérale de Lausanne, Switzerland, 1995.

6. Sam-Haroud D. and Faltings B.V. Consistency techniques for continuous constraints. In Constraints Journal, 1, 1996.

7. Samet H. Applications of spatial data structures - Computer graphics, image processing and GIS. Addison-Wesley Publishing Company, 1993.

8. van Beek P. On the minimality and decomposability of constraint networks. In Proceedings of the 10th National Conference on AI, 1992.

9. van Beek P. and Dechter R. On the minimality and global consistency of row convex constraint networks. In Journal of the ACM, 1995.

This article was processed using the $\mathrm{IAT}_{\mathrm{E}} \mathrm{X}$ macro package with LLNCS style 